\title{
Superconductivity and other phases in graphene multilayers, without twists
}

1. Isospin magnetism and spin-triplet superconductivity in Bernal bilayer graphene

Authors: Haoxin Zhou, Yu Saito, Liam Cohen, William Huynh, Caitlin L. Patterson, Fangyuan Yang, Takashi Taniguchi, Kenji Watanabe, Andrea F. Young arXiv:2110.11317

2. Superconductivity in rhombohedral trilayer graphene Authors: Haoxin Zhou, Tian Xie, Takashi Taniguchi, Kenji Watanabe, Andrea F. Young arXiv:2106.07640

3. Cascade of isospin phase transitions in Bernal bilayer graphene at zero magnetic field Authors: Sergio C. de la Barrera, Samuel Aronson, Zhiren Zheng, Kenji Watanabe, Takashi Taniguchi, Qiong Ma, Pablo Jarillo-Herrero, Raymond Ashoori arXiv:2110.13907

4. Quantum cascade of new correlated phases in trigonally warped bilayer graphene Authors: Anna M. Seiler, Fabian R. Geisenhof, Felix Winterer, Kenji Watanabe, Takashi Taniguchi, Tianyi Xu, Fan Zhang, R. Thomas Weitz arXiv:2111.06413

Recommended with a Commentary by Francisco Guinea, Imdea Nanoscience, Madrid, Spain, and Donostia International Physics Center, San Sebastian, Spain

The last commentary posted by me in this journal club[1] started with the sentence: The phase diagram of twisted bilayer graphene keeps bringing up surprises. The present commentary can well begin with the same sentence, except that the word twisted is to be removed. Recent experiments have shown superconductivity and correlated phases in graphene trilayers[2], and also in bilayer graphene[3] (superconductivity and correlated phases), and $[4,5]$ (correlated phases). The experiments have been carried out on systems already studied in the past, and they suggest that carbon compounds admit a non trivial phase diagram without the existence of a complex moiré pattern. 


\section{Phase diagram of a graphene bilayer}

We consider first the experiments on bilayer graphene[3, 4, 5], as this is the system most extensively studied, both theoretically, and experimentally.

The electronic structure of bilayer graphene can be understood in terms of a simple extension of the Dirac model used for monolayer graphene[6]. The hopping between layers changes significantly the Dirac cones, and, to a first approximation, these cones are replaced by two parabolic bands which touch at half filling. Quantum Hall measurements confirmed rapidly the validity of this model[7]. The parabolic band touching implied that the charge susceptibility, as well as other susceptibilities had a logarithmic divergence at half filling[8]. This divergence, analyzed with renormalization group methods and other techniques leads to a variety of possible correlated phases, including superconductivity (see[9, 10, 11, 12], and also the review[13]). A perpendicular electric field shifts the voltage difference between the two layers, leading to a gap, and to bands with a "mexican hat" dispersion[13], which implies a divergent density of states at the gap edges. A number of experiments reported correlated behavior in graphene bilayers[14, 15, 16, 17, 18], although the evidence sometimes admitted alternative explanations[19].

The experiments in $[3,4,5]$ analyze in great detail the phase diagram of bilayer graphene. Ref.[5] use transport measurements to probe the system. Ref.[4] focuses on results for the electronic compressibility, and ref.[3] presents both transport and compressibility measurements. In the three references, the effects of changes in the number of carriers, applied electric field, and magnetic field are studied.

A common finding in these works $[3,4,5]$ is the existence of flavor (spin or valley) polarized metallic phases. Flavor polarization of the carriers changes the number of pockets of the Fermi surface in the Brillouin Zone. This effect, in turn, alters the degeneracy of the Landau levels induced by a perpendicular magnetic field. By counting the number of electrons needed to fill up the Landau levels at a given energy, the number of pockets at the Fermi surface can be inferred. In a similar fashion, the oscillations of the conductivity at low magnetic fields provides information on the size of the Fermi surface, and on the number of carriers that it encompasses. Refs. [3, 4, 5] use variations of this analysis to show that, for certain densities and values of the electric field, the number of Fermi surface pockets is less than four, so that the system is valley and/or spin polarized. In order to identify a spin from a valley polarized phase, ref.[4] use an in plane magnetic field, which only couples to the spin degree of freedom. The fact that the field modifies the phase boundaries implies that there are spin polarized phases. Ref.[3] identifies a spin polarized phase from its proximity to a spin polarized superconducting phase (see below), and ref.[5] relates magnetic phases to observed hysteretic behavior. This succession of metallic flavor polarized phase resembles the "cascade" of phase transitions previously found in twisted bilayer graphene[20, 21]. Recent theoretical calculations support this picture[22].

Ref.[5] also reports insulating phases which show anomalous Hall features, as a quantized longitudinal conductivity was found for very low perpendicular magnetic fields. Moreover, in some phases, the quantization of the Hall conductance does not show the values expected in a pure Quantum Anomalous Hall phase (QAH), suggesting that the phase is a combination of of a QAH and a Wigner crystal[23].

Finally, [3] presents evidence for superconductivity, with an estimated 2D critical tem- 
perature of $T_{B K T} \approx 26 \mathrm{mK}$. This phase occurs in the vicinity of a flavor polarized phase, for a carrier density $n \approx-0.57 \times 10^{12} \mathrm{~cm}^{-2}$ and electric field $D \approx 1.02 \mathrm{~V} / \mathrm{nm}$. Superconductivity persists up to fields $B_{\|} \gtrsim 0.16 \mathrm{~T}$. The magnitude of these fields, as compared to the value of the critical temperature, strongly suggests spin triplet order pairing. The critical field allows to estimate the coherence length, $\xi \approx 250 \mathrm{~nm}$. Normal disorder suppresses superconductivity in superconductors where the order parameter changes sign over the Fermi surface[24]. The system studied in ref.[3] has an elastic mean free path, $\ell \sim 5 \mu \mathrm{m}$, comparable to the sample size. As $\ell \gg \xi$ pair breaking effects are expected to be small.

\section{Phase diagram of a rhombohedral graphene trilayer}

Ref.[2] has studied the phase diagram of an $A B C$ trilayer graphene. The $A B C$ stacking order is a metastable arrangement of graphene layers. The relative orientation of nearest neighbor layers is the same as in the $A B A$ Bernal arrangement, and the energy difference between the rhombohedral and Bernal arrangements is small (a recent calculation[25] suggests that the ABC stacking might be the most stable trilayer). The infinite stack $\cdots A B C A B C \cdots$, defined as rhombohedral graphite, has been studied in the past[26, 27]. Within a simple approximation, the electronic structure can be reduced to a set of Schrieffer$\mathrm{Su}$-Heeger models[28], one for each value of $k_{z}$, where $k_{z}$ is the wavevector perpendicular to the layers. This analysis shows the existence of surface states for a finite set of $\vec{k}_{\|}$values, showing that rhomohedral graphite is, in modern terms, a Dirac semimetal[29]. Rhomobohedral inclusions are expected in graphite samples with stacking disorder, pyrolytic graphite. The electronic structure of rhombohedral multilayers has been investigated, showing the expected enhancement of the density of states at the surface layers[30, 31]. Some evidence for correlated behavior in these systems was already reported in $[32,17,34,35,36]$. The lab which produced ref.[2] had previously published a detailed analysis of correlated phases (although not superconductivity) in[37].

The large surface density of states in rhombohedral graphite has lead to the proposal that this three dimensional system can be a high- $T_{c}$ superconductor[38, 39]. In fact, some evidence of superconductivity in graphite samples, possibly with stacking defects within them, has been reported[40].

Ref.[2] reports transport experiments in $A B C$ trilayer graphene, as function of carrier density, perpendicular electric field, and magnetic field (previous experiments reporting superconductivity in a similar system were presented in[41]). The results show two superconducting regions, labeled SC1 and SC2. These phases have different critical temperatures and fields, $T_{c} \approx 106 \mathrm{mK}, B_{c} \approx 10 \mathrm{mT}$ for SC1, and $T_{c} \lesssim 20 \mathrm{mK}, B_{c} \approx 1 \mathrm{mT}$ for SC2 (note that, in this phase, the resistivity does not drop all the way to zero at the lowest temperatures probed). The experiments do not indicate the existence of insulating phases. Quantum oscillations induced by the magnetic field, which are sensitive to the size of the Fermi surface, suggest the existence of a variety of phases: fully polarized, partial spin or valley polarized, and not polarized. Phase SC1 appears near the partially polarized phase, at a displacement field $D \approx 0.5 \mathrm{~V} \mathrm{~nm}^{-1}$. and a carrier density $n \approx 2 \times 10^{12} \mathrm{~cm}^{-1}$. From the value of $B_{c}$ the coherence length of phase SC1 is estimated to be $\xi \approx 150-200 \mathrm{~nm}$, while normal state resistivity measurements suggest an elastic mean free path $\ell \approx 1 \mu \mathrm{m}$. Finally, the relation between $B_{c}$ and $T c$ is consistent with singlet pairs. By contrast, features associated to phase 
SC2 persist up to magnetic fields of order of 1T, suggesting spin triplet superconductivity.

The experiments reported in[2] have led to the proposal of a variety of models for the superconducting phases $[42,43,44,45,46,47,48]$. The models can be roughly classified into pairing mediated by electron-phonon coupling[42], pairing due to excitations from a nearby broken symmetry phase[43, 44, 46], or pairing arising from repulsive interactions [45, 47, 48] (or, alternatively, damped charge fluctuations[45, 47]).

\section{Outlook}

The finding of correlated phases and superconductivity in graphene systems without a moiré superstructure was not widely expected. Graphite intercalation compounds[27], and doped fullerene crystals[49] were the only carbon compounds which showed a robust superconductivity, although at carrier concentrations at least two orders of magnitude larger than those found in the experiments commented here, $n \sim 10^{14} \mathrm{~cm}^{-2}$.

A crucial open question is the similarities, and differences, between the phase diagrams reported in $[2,3,4,5]$ and those observed in twisted bilayer and trilayer graphene[50, 51, 52]. Superconductivity and other correlated phases in a Bernal graphene bilayers, and in rhombohedral trilayers are observed at temperatures at least one order of magnitude lower than the temperatures needed to probe twisted systems. It could happen that the correlated phases are induced by different physical processes in twisted and non twisted arrangements, despite the fact that the electronic structure is solely determined by simple carbon $p_{z}$ orbitals.

Lastly, the number of possible combinations of graphene layers is enormous. The quasi2D nature of these compounds, and the existence of the (chiral) valley degree of freedom allow for broken symmetry phases not found in other materials. Already the phase diagram of twisted bilayer graphene shares features with strongly correlated materials and with 2D electron gases in the Quantum Hall regime. The observations in $[2,3,4,5]$ make it plausible that many of these graphene stacks will host unusual phases. It seems likely that the search for new physics in carbon compounds is still in its early stages.

\section{References}

[1] F. Guinea, DOI: 10.36471/JCCM, November 202001.

[2] Haoxin Zhou, Tian Xie, Takashi Taniguchi, Kenji Watanabe, Andrea F. Young, Superconductivity in rhombohedral trilayer graphene, arXiv:2106.07640, Nature 598, (2021) (2021).

[3] Haoxin Zhou, Yu Saito, Liam Cohen, William Huynh, Caitlin L. Patterson, Fangyuan Yang, Takashi Taniguchi, Kenji Watanabe, Andrea F. Young, Isospin magnetism and spin-triplet superconductivity in Bernal bilayer graphene, arXiv:2110.11317.

[4] Sergio C. de la Barrera, Samuel Aronson, Zhiren Zheng, Kenji Watanabe, Takashi Taniguchi, Qiong Ma, Pablo Jarillo-Herrero, Raymond Ashoori, Cascade of isospin phase transitions in Bernal bilayer graphene at zero magnetic field, arXiv:2110.13907 (2021). 
[5] Anna M. Seiler, Fabian R. Geisenhof, Felix Winterer, Kenji Watanabe, Takashi Taniguchi, Tianyi Xu, Fan Zhang, R. Thomas Weitz, Quantum cascade of new correlated phases in trigonally warped bilayer graphene, arXiv:2111.06413 (2021).

[6] E. McCann, V. I Fal'ko, Landau level degeneracy and quantum Hall effect in a graphite bilayer, Phys. Rev. Lett. 96, 086805 (2006).

[7] K. S. Novoselov, E. McCann, S. V. Morozov, V. I. Falko, M. I. Katsnelson, U. Zeitler, D. Jiang, F. Schedin, A. K. Geim, Unconventional quantum Hall effect and Berry's phase of $2 \pi$ in bilayer graphene, Nature Phys. 2, 177 (2006).

[8] Johan Nilsson, A. H. Castro Neto, N. M. R. Peres, F. Guinea, Electron-electron interactions and the phase diagram of a graphene bilayer, Pys. Rev. B 73, 214418 (2006).

[9] Hongki Min, Giovanni Borghi, Marco Polini, A.H. MacDonald, Pseudospin Magnetism in Graphene, Phys. Rev. B 77, 041407(R) (2008).

[10] O. Vafek, Interacting fermions on the honeycomb bilayer: From weak to strong coupling, Phys. Rev. B 82, 205106 (2010).

[11] Rahul Nandkishore, Leonid Levitov, Dynamical Screening and Excitonic Instability in Bilayer Graphene, Phys. Rev. Lett. 104, 156803 (2010).

[12] Y. Lemonik, I. L. Aleiner, V. I. Fal'ko, Competing Nematic, Anti-ferromagnetic and Spin-flux orders in the Ground State of Bilayer Graphene, Phys. Rev. B 85, 245451 (2012).

[13] Edward McCann, Mikito Koshino, The electronic properties of bilayer graphene, Rep. Prog. Phys. 73, 057503 (2013).

[14] R. Thomas Weitz, Monica T. Allen, Benjamin E. Feldman, Jens Martin, Amir Yacoby, Coulomb-driven broken-symmetry states in doubly gated suspended bilayer graphene, Science 330, 812 (2010).

[15] F. Freitag, J. Trbovic, M. Weiss, C. Schönenberger, Spontaneously gapped ground state in suspended bilayer graphene, Phys. Rev. Lett. 108, 076602 (2012).

[16] Wenzhong Bao, Jairo Velasco Jr, Fan Zhang, Lei Jing, Brian Standley, Dmitry Smirnov, Marc Bockrath, Allan MacDonald, Chun Ning Lau, Minimum Conductivity and Evidence for Phase Transitions in Ultra-clean Bilayer Graphene, Proc. Nat. Acad. Sc. (USA) 109, 10802 (2012).

[17] Kayoung Lee, Babak Fallahazad, Jiamin Xue, David C. Dillen, Kyounghwan Kim, Takashi Taniguchi, Kenji Watanabe, Emanuel Tutuc, Chemical Potential and Quantum Hall Ferromagnetism in Bilayer Graphene, cience 345, 58 (2014).

[18] Fabian R. Geisenhof, Felix Winterer, Anna M. Seiler, Jakob Lenz, Tianyi Xu, Fan Zhang, R. Thomas Weitz, Tunable quantum anomalous Hall octet driven by orbital magnetism in bilayer graphene, Nature 598, 53 (2021). 
[19] P. San-Jose, R. V. Gorbachev, A. K. Geim, K. S. Novoselov, F. Guinea, Stacking boundaries and transport in bilayer graphene, Nano Lett. 14, 2052 (2014).

[20] Dillon Wong, Kevin P. Nuckolls, Myungchul Oh, Biao Lian, Yonglong Xie, Sangjun Jeon, Kenji Watanabe, Takashi Taniguchi, B. Andrei Bernevig, Ali Yazdani, Cascade of electronic transitions in magic-angle twisted bilayer graphene, arXiv:1912.06145, Nature 582, 198 (2020), arXiv:1912.06145.

[21] Uri Zondiner, Asaf Rozen, Daniel Rodan-Legrain, Yuan Cao, Raquel Queiroz, Takashi Taniguchi, Kenji Watanabe, Yuval Oreg, Felix von Oppen, Ady Stern, Erez Berg, Pablo Jarillo-Herrero, Shahal Ilani, Cascade of Phase Transitions and Dirac Revivals in Magic Angle Graphene, Nature, 582, 203 (2020), arXiv:1912.06150.

[22] Zhiyu Dong, Margarita Davydova, Olumakinde Ogunnaike, Leonid Levitov, Isospin ferromagnetism and momentum polarization in bilayer graphene, arXiv:2110.15254 (2021).

[23] Z. Tešanović, F. Axel, B. I. Halperin, "Hall crystal" versus Wigner crystal, Phys. Rev. B 39, 8525 (1989).

[24] Note that disorder in graphene leads to long range (intravalley) and short range (intervalley) scattering. In a spin triplet/valley singlet gapped superconductor, only short range scattering is pair breaking.

[25] Raúl Guerrero-Avilés, Marta Pelc, Fabian Geisenhof, Thomas Weitz, Andrés Ayuela, Relative Stability of Bernal and Rhombohedral Stackings in Trilayer Graphene under Distortions, arXiv:2110.06590 (2021).

[26] J. W. McClure, Electron energy band structure and electronic properties of rhombohedral graphite, Carbon 7, 425 (1969).

[27] M. S. Dresselhaus and G. Dresselhaus, Intercalation compounds of graphite, Adv. in Phys. 51, 1 (2002).

[28] D. P. Arovas and F. Guinea, Stacking faults, bound states, and quantum hall plateaus in crystalline graphite, Phys. Rev. B 78, 245416 (2008).

[29] N.P. Armitage, E. J. Mele, Ashvin Vishwanath, Weyl and Dirac Semimetals in Three Dimensional Solids, Rev. Mod. Phys. 90, 015001 (2018).

[30] F. Zhang, B. Sahu, H. Min, and A. H. MacDonald, Band structure of abc-stacked graphene trilayers, Phys. Rev. B 82, 035409 (2010).

[31] M. Koshino, Interlayer screening effect in graphene multilayers with aba and abc stacking, Phys. Rev. B 81, 125304 (2010).

[32] W. Bao, L. Jing, J. Velasco Jr., Y. Lee, G. Liu, and Tran, B. Standley, M. Aykol, S. B. Cronin, D. Smirnov, M. Koshino, E. McCann, M. Bockrath, and C. N. Lau, Stacking-dependent band gap and quantum transport in trilayer graphene, Nature Phys. 7, 948 (2011). 
[33] Y. Lee, D. Tran, K. Myhro, J. Velasco, N. Gillgren, C. N. Lau, Y. Barlas, J. M. Poumirol, D. Smirnov, and F. Guinea, Competition between spontaneous symmetry breaking and single-particle gaps in trilayer graphene, Nature Comm. 5, 5656 (2014).

[34] G. Chen, L. Jiang, S. Wu, B. Lyu, H. Li, B. L. Chittari, K. Watanabe, T. Taniguchi, Z. Shi, J. Jung, Y. Zhang, and F. Wang, Evidence of a gate-tunable mott insulator in a trilayer graphene moir superlattice, Nature Phys. 15, 237 (2019).

[35] Y. Lee, S. Che, J. Velasco Jr., D. Tran, J. Baima, F. Mauri, M. Calandra, M. Bockrath, and C. N. Lau, Gate tunable magnetism and giant magnetoresistance in abcstacked few-layer graphene, arXiv:1911.04450 (1919).

[36] Y. Shi, S. Xu, Y. Yang, S. Slizovskiy, S. V. Morozov, S.-K. Son, S. Ozdemir, C. Mullan, J. Barrier, J. Yin, A. I. Berdyugin, B. A. Piot, T. Taniguchi, K. Watanabe, V. I. Falko, K. S. Novoselov, A. K. Geim, and A. Mishchenko, Electronic phase separation in multilayer rhombohedral graphite, Nature 584, 210 (2020).

[37] Haoxin Zhou, Tian Xie, Areg Ghazaryan, Tobias Holder, James R. Ehrets, Eric M. Spanton, Takashi Taniguchi, Kenji Watanabe, Erez Berg, Maksym Serbyn, Andrea F. Young, Half and quarter metals in rhombohedral trilayer graphene, Nature 598, 429 (2021).

[38] N. B. Kopnin, T. T. Heikkilä, and G. E. Volovik, High-temperature surface superconductivity in topological flat-band systems, Phys. Rev. B 83, 220503 (2011).

[39] N. B. Kopnin, M. Ijäs, A. Harju, and T. T. Heikkilä, High-temperature surface superconductivity in rhombohedral graphite, Phys. Rev. B 87, 140503 (2013).

[40] Pablo D. Esquinazi, Ordered Defects: A Roadmap towards room temperature Superconductivity and Magnetic Order, arXiv:1902.07489 (2019).

[41] G. Chen, A. L. Sharpe, P. Gallagher, I. T. Rosen, E. J. Fox, L. Jiang, B. Lyu, H. Li, K. Watanabe, T. Taniguchi, J. Jung, Z. Shi, D. Goldhaber-Gordon, Y. Zhang, and F. Wang, Signatures of tunable superconductivity in a trilayer graphene moiré superlattice, Nature 572, 215 (2019).

[42] Y.-Z. Chou, F. Wu, J. D. Sau, and S. Das Sarma, Acoustic-phonon-mediated superconductivity in rhombohedral trilayer graphene, Phys. Rev. Lett. 127, 187001 (2021).

[43] H. Dai, J. Hou, X. Zhang, Y. Liang, and T. Ma, Mott insulating state and d +i d superconductivity in an ABC graphene trilayer, Phys. Rev. B 104, 035104 (2021).

[44] Z. Dong and L. Levitov, Superconductivity in the vicinity of an isospin-polarized state in a cubic Dirac band, arXiv:2109.01133 (2021). 
[45] A. Ghazaryan, T. Holder, S. Maksym, and E. Berg, Un-conventional superconductivity in systems with annular fermi surfaces: Application to rhombohedral trilayer graphene, arXiv:2109.00011 (2021).

[46] S. Chatterjee, T. Wang, E. Berg, and M. P. Zaletel, Inter-valley coherent order and isospin fluctuation mediated superconductivity in rhombohedral trilayer graphene, arXiv:2109.00002 (2021).

[47] Tommaso Cea, Pierre A. Pantaleón, Vo Tien Phong, Francisco Guinea, Superconductivity from Repulsive Interactions in Rhombohedral Trilayer Graphene: a Kohn-Luttinger-Like Mechanism, arXiv:2109.04345 (2021).

[48] Yi-Zhuang You, Ashvin Vishwanath, Kohn-Luttinger Superconductivity and Inter-Valley Coherence in Rhombohedral Trilayer Graphene, arXiv:2109.04669 (2021).

[49] A. F. Hebard, Superconductivity in doped fullerenes., Physics Today 45, 26 (1992).

[50] Yuan Cao, Valla Fatemi, Shiang Fang, Kenji Watanabe, Takashi Taniguchi, Efthimios Kaxiras, Pablo Jarillo-Herrero, Magic-angle graphene superlattices: a new platform for unconventional superconductivity Nature, 556, 43 (2018).

[51] Jeong Min Park, Yuan Cao, Kenji Watanabe, Takashi Taniguchi, Pablo Jarillo-Herrero, Tunable Phase Boundaries and Ultra-Strong Coupling Superconductivity in Mirror Symmetric Magic-Angle Trilayer Graphene Nature 590, 249 (2021).

[52] Zeyu Hao, A. M. Zimmerman, Patrick Ledwith, Eslam Khalaf, Danial Haie Najafabadi, Kenji Watanabe, Takashi Taniguchi, Ashvin Vishwanath, Philip Kim, Electric field tunable unconventional superconductivity in alternating twist magic-angle trilayer graphene, Science 371, 1133 (2021). 